\title{
ZERO DISSIPATION AND STABILITY OF SHOCKS
}

\author{
Tai-Ping Liu
}

\begin{abstract}
We study the nonlinear stability of viscous shock waves in the process of vanishing viscosity parameters. We reason with the conservation laws and the time-asymptotic states that the two types of non-classical shocks, overcompressive and undercompressive shocks, are nonlinearly stable in different senses. For overcompressive shock waves, the time-asymptotic state depends on the strength of the viscosity. In particular, the overcompressive shocks are nonlinearly stable, but not uniformly with respect to the strength of the viscosity. We show this for a rotationally invariant system derived from magneto-hydrodynamics and nonlinear elasticity. On the other hand, the existence and speed of undercompressive shocks depend sensitively on the relative strength of the dissipation parameters. Nevertheless, these waves are nonlinearly stable uniformly with respect to the strength of the dissipations. We demonstrate this for a combustion model. These phenomena for overcompressive and undercompressive shocks hold for general systems and are in marked contrast to the classical shocks for gas dynamics.
\end{abstract}

\section{Introduction}

Consider the hyperbolic conservation laws

$$
u_{t}+f(u)_{x}=0 .
$$

It is well known that, due to the nonlinearity of the flux function $f(u)$, its solutions contains shock waves in general. One of the basic issues for shock wave theory is to decide the admissibility of the weak solutions. This is done by prescribing the entropy condition for the shocks [9], [10]. A canonical way to find the condition is to apply the viscosity criterion. The criterion states that a shock for the hyperbolic conservation laws is admissible if there exists a corresponding traveling wave, the viscous shock wave, for the associated viscous conservation laws

$$
u_{t}+f(u)_{x}=\left(B(u) u_{x}\right)_{x}
$$

with a physically relevant viscosity matrix $B(u)$. This is reasonable since the viscous conservation laws are physically more complete, and a viscous shock converges to a discontinuous inviscid shock as the viscosity matrix $B(u)$ tends to zero. The process works for the important gas dynamics equations where the hyperbolic system is the compressible Euler equations and the viscous system is the compressible Navier-Stokes equations [1]. However, the process fails when physical effects besides compression and convection are present, as for the magneto-hydrodynamics equations, full nonlinear elasticity equations, multi-phase flows, and combustion models.

Received April 16, 1997, revised October 28, 1997.

1991 Mathematics Subject Classification: 35L65.

Key words and phrases: conservation laws, zero dissipation, viscous shock waves, overcompressive shock, undercompressive shock. 
There are two questions one may then ask: Is it always possible to have a physically relevant well-posed hyperbolic theory? If so, how much is inherited and/or lost in the process of zero dissipation?

For the gas dynamics equations, as we noted already, the answer to the first question is yes after the efforts of Riemann, Stokes, Rankine, Hugoniot, Prandtl, Taylor, and others [1]. The answer to the second question is that, except for the structure of viscous layers, nothing is lost. In other words, the solutions of the viscous conservation laws converge to those of the hyperbolic conservation laws uniformly away from the shock, initial, and boundary layers. The wave patterns evolve smoothly in the zero dissipation limit. Although rigorous analysis of this fact is still wanting, it is generally believed and has been checked for some class of solutions, cf. [6], [7]. What characterizes the gas dynamics equations is that its inviscid shock is stable in the linearized sense. To see this we take the simplest case, the isentropic Euler equations in the Lagrangian coordinates

$$
\begin{gathered}
\tau_{t}-v_{t}=0, \\
v_{t}+p(\tau)_{x}=0 .
\end{gathered}
$$

Here $\tau, v$, and $p=p(\tau)$ are, respectively, the specific volume, velocity, and the pressure of the gas. The system is strictly hyperbolic with characteristic speeds

$$
\lambda_{1}=-\sqrt{p^{\prime}(\tau)}, \quad \lambda_{2}=\sqrt{p^{\prime}(\tau)} .
$$

A shock $\left(\tau_{-}, v_{-} ; \tau_{+}, v_{+}\right)$with speed $s$ satisfies the Rankine-Hugoniot condition

$$
s\left(\tau_{+}-\tau_{-}\right)=-\left(v_{+}-v_{-}\right), \quad s\left(v_{+}-v_{-}\right)=p\left(\tau_{+}\right)-p\left(\tau_{-}\right),
$$

by the weak formulation of the equations. When $\lambda_{1}$ compresses to form a 1-shock, it satisfies the entropy condition

$$
\lambda_{1}\left(\tau_{+}\right)<s<\lambda_{1}\left(\tau_{-}\right), \quad s<\lambda_{2}\left(\tau_{ \pm}\right) .
$$

Similarly, a 2-shock satisfies the entropy condition

$$
\lambda_{1}\left(\tau_{ \pm}\right)<s, \quad \lambda_{2}\left(\tau_{+}\right)<s<\lambda_{2}\left(\tau_{+}\right) .
$$

In either case, three characteristics impinge on the shock while one leaves it. For a 1-shock, the eigen direction $r_{2}\left(\tau_{+}, v_{+}\right)$for the leaving characteristic $\lambda_{2}\left(\tau_{+}\right)$and the shock jump $\left(\tau_{+}-\tau_{-}, v_{+}-v_{-}\right)$are linearly independent. Here $r_{i}, i=1,2$, are the right eigenvectors for the gradient of the flux function. Similarly, linear independence also holds for the 2-shocks. With these, the shocks are linearly stable.

This important criterion of linear stability is generalized to a general system of hyperbolic conservation laws [9]. We call such a shock the classical Lax-shock.

For the magneto-hydrodynamics equations, there has been the problem of the admissibility of intermediate shocks [8]. Such a shock is admissible according to the viscosity criterion. On the other hand, admitting such a shock would make the hyperbolic system not well-posed; there is more than one solution to some Riemann problem. In the present article, we study this issue for the following rotationally invariant model

$$
\begin{gathered}
u_{t}+\left(u\left(u^{2}+v^{2}\right)\right)_{x}=\varepsilon u_{x x} \\
v_{t}+\left(v\left(u^{2}+v^{2}\right)\right)_{x}=\varepsilon v_{x x}
\end{gathered}
$$


Here $\varepsilon$ is a positive constant representing the strength of the viscosity, and $u, v$ are both scalar functions. The model is derived from the magneto-hydrodynamics equations modeling the interaction of shocks and Alfven's waves, see [2] and references therein. One of the important differences between this system and the gas dynamics equations is that the corresponding inviscid system for (1.1) is not strictly hyperbolic, and that there exist shock waves for which all four characteristics on either side of the shock impinge on the shock. Such a shock is more compressive than a gas dynamics shock and is therefore called an overcompressive shock. There also are shocks which are classical Laxian in terms of the number of characteristics impinging on the shock, but fail to satisfy the linear independence requirement. The study of these two types of shocks, particularly their nonlinear stability in the process of zero dissipation, is the main purpose of the first half of the present article. We will analyze the conservation laws

$$
\begin{aligned}
& \frac{d}{d t} \int_{-\infty}^{\infty} u(x, t) d x=0 \\
& \frac{d}{d t} \int_{-\infty}^{\infty} v(x, t) d x=0
\end{aligned}
$$

particularly in the process of zero dissipation. We show that an overcompressive shock is nonlinearly stable, but splits into two waves of different speeds when the viscosity is small compared with the perturbation. The shocks without the linear independence condition always split when perturbed. Some of these results have been reported without proof in [11].

Waves which are less compressive than the classical Lax shocks occur in several important physical situations such as combustion and multi-phase flow. These undercompressive shocks are nonlinearly stable and are hyperbolic waves. However, the shape and speed of these waves depend sensitively on the relative strengths of dissipation parameters. Consequently, there is more than one hyperbolic theory, depending on the relative strength of the dissipation parameters. We illustrate this for the combustion model

$$
\begin{gathered}
(u+q z)_{t}+f(u)_{x}=\alpha u_{x x} \\
z_{t}=K \phi(u) z
\end{gathered}
$$

see [4], [15], and references therein. A more realistic model can be found in [5], [16]. However, these models share the same qualitative behavior. For analytical studies of stability of classical shocks, see [12], and for non-classical shocks for fixed viscosity, see [3], [14], and references therein.

\section{Part I. Overcompressive shocks}

\section{Hyperbolic theory}

Consider the inviscid system associated with (1.1)

$$
\begin{aligned}
& u_{t}+\left(u\left(u^{2}+v^{2}\right)\right)_{x}=0 \\
& v_{t}+\left(v\left(u^{2}+v^{2}\right)\right)_{x}=0
\end{aligned}
$$




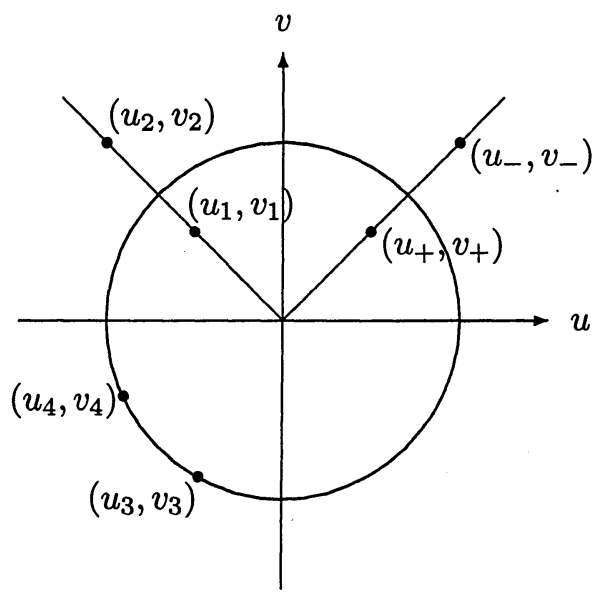

FIGURE 2.1

The eigenvectors and eigenvalues are

$$
\begin{gathered}
r_{1}(u, v)=(v,-u), \quad r_{2}(u, v)=(u, v), \\
\lambda_{1}(u, v)=u^{2}+v^{2}, \quad \lambda_{2}(u, v)=3 \lambda_{1}(u, v) .
\end{gathered}
$$

The 1-characteristic is linearly degenerate, and the 2-characteristic is genuinely nonlinear except at the origin

$$
\nabla \lambda_{1} \cdot r_{1}(u, v)=0, \quad \nabla \lambda_{2} \cdot r_{2}(u, v)=6\left(u^{2}+v^{2}\right)
$$

Thus a shock wave has end states along the same radial direction through the origin, i.e., in the direction of $r_{2}(u, v)$. If we confine ourself to the classical Lax shocks, then a shock $\left(u_{-}, v_{-} ; u_{+}, v_{+}\right)$has the property that $u_{-} v_{+}=u_{+} v_{-}, u_{-} u_{+} \geq 0, v_{-} v_{+} \geq 0$. A rarefaction wave $\left(u_{1}, v_{1} ; u_{2}, v_{2}\right)$ takes values along the same lines but in the opposite direction, and a contact discontinuity $\left(u_{3}, v_{3} ; u_{4}, v_{4}\right)$ takes values along a circle $u^{2}+$ $v^{2}=$ constant, Figure 2.1. The Riemann problem (2.1) with

$$
(u, v)(x, 0)= \begin{cases}\left(u_{l}, v_{l}\right), & x<0 \\ \left(u_{r}, v_{r}\right), & x>0\end{cases}
$$

can be solved by a contact discontinuity $\left(u_{l}, v_{l} ; u_{m}, v_{m}\right)$ and either a shock, Figure 2.2, or a rarefaction wave $\left(u_{m}, v_{m} ; u_{r}, v_{r}\right)$, Figure 2.3.

In the above solution of the Riemann problem, shock waves crossing the origin $(u, v)=(0,0)$ are not included. Some of these shocks are overcompressive and may be viewed as the combination of a Lax shock and a contact discontinuity and is called an intermediate shock in magneto-hydrodynamics. The Riemann problem would have more than one solution if intermediate shocks are regarded as admissible, Figures 2.2 and 2.4 . 

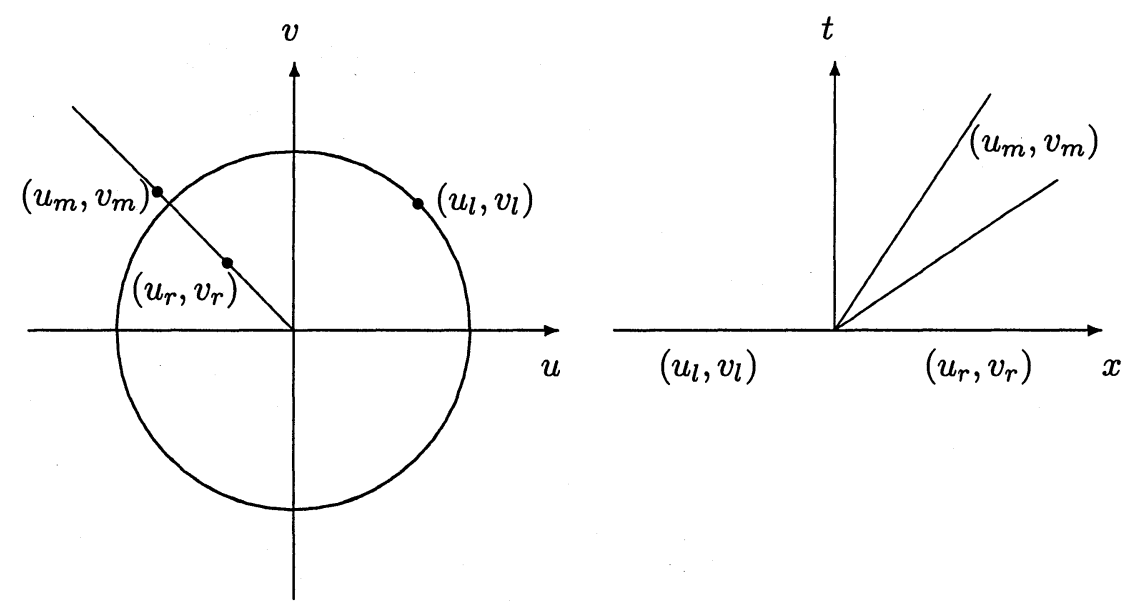

FIGURE 2.2

\section{Viscous profiles}

We now consider the traveling waves for the viscous system (1.1):

$$
\begin{gathered}
\phi^{\prime}(\xi)=-s\left(\phi-u_{-}\right)+\phi\left(\phi^{2}+\psi^{2}\right)+A \\
\psi^{\prime}(\xi)=-s\left(\psi-v_{-}\right)+\psi\left(\phi^{2}+\psi^{2}\right)+B \\
(u, v)(x, t)=(\phi, \psi)(\xi), \quad \xi=(x-s t) / \varepsilon, \quad(\phi, \psi)( \pm \infty)=\left(u_{ \pm}, v_{ \pm}\right),
\end{gathered}
$$

where we have integrated (1.1) with the integration constants

$$
\begin{gathered}
A=-u_{-}\left(u_{-}{ }^{2}+v_{-}{ }^{2}\right)=s\left(u_{+}-u_{-}\right)-u_{+}\left(u_{+}{ }^{2}+v_{+}{ }^{2}\right), \\
B=-v_{-}\left(u_{-}{ }^{2}+v_{+}{ }^{2}\right)=s\left(v_{+}-v_{-}\right)-v_{+}\left({u_{+}}^{2}+v_{+}{ }^{2}\right) .
\end{gathered}
$$
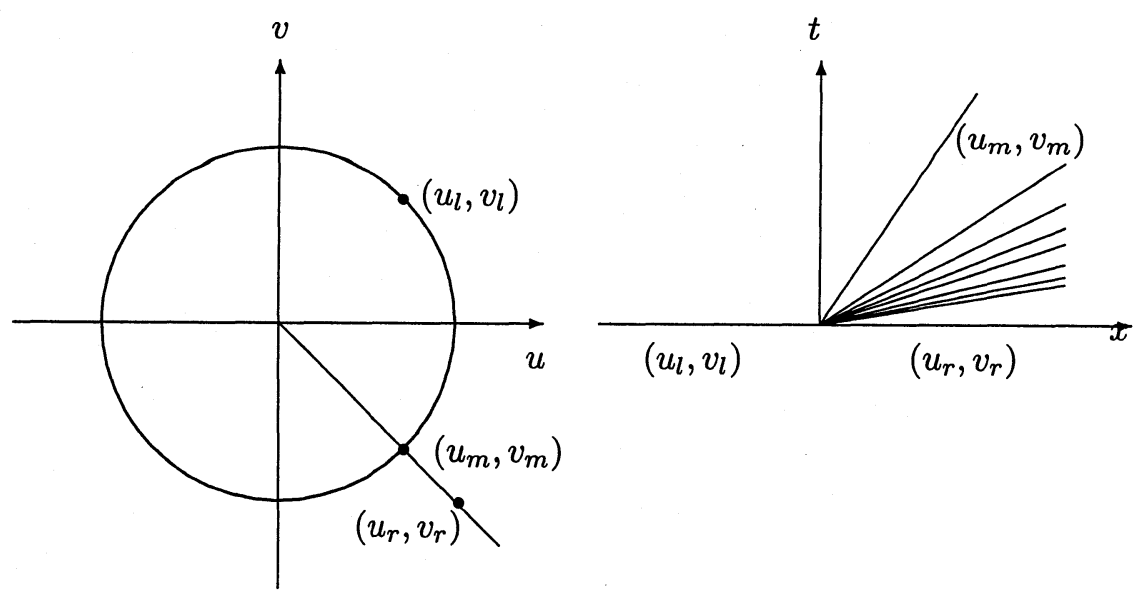

FigURE 2.3 


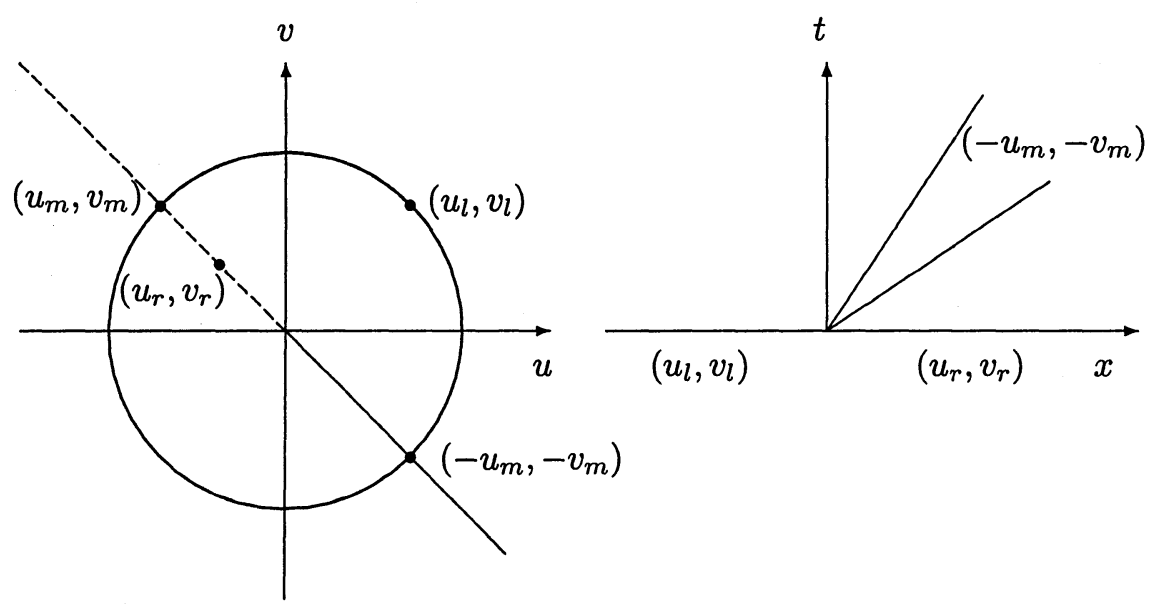

FigURE 2.4

For a classical Lax shock, Figure 2.2, there exists a unique saddle-node connection. We are interested in shocks which cross the origin, that is, the end states $\left(u_{-}, v_{-}\right)$and $\left(u_{+}, v_{+}\right)$lie on the opposite sides of the origin. Since the system (1.1) is rotationally invariant, without loss of generality, we take

$$
\left(u_{-}, v_{-}\right)=\left(u_{-}, 0\right), \quad\left(u_{+}, v_{+}\right)=\left(u_{+}, 0\right), \quad u_{+}<0<u_{-} .
$$

It is easy to see that connecting orbits exist if, Figure 3.1,

$$
0>u_{+}>u_{0}, \quad 3 u_{0}^{2} \equiv \frac{u_{0}^{3}-u_{-}^{3}}{u_{0}-u_{-}} .
$$

With (3.2), the shock is overcompressive

$$
\lambda_{i}\left(u_{+}, 0\right)<s<\lambda_{i}\left(u_{-}, 0\right), \quad i=1,2 .
$$

This implies that the critical point $\left(u_{-}, 0\right)$ for the ODE (3.1) is an unstable node and $\left(u_{+}, 0\right)$ is a stable node. As a consequence, there is a 1-parameter family of connecting trajectories, Figure 3.2. These orbits are bounded by the two orbits $\pm\left(\phi_{*}, \psi_{*}\right)$ connecting $\left(u_{-}, 0\right)$ and $\left(u_{*}, 0\right)$, with

$$
\frac{u_{*}^{3}-u_{-}^{3}}{u_{*}-u_{-}} \equiv \frac{u_{+}^{3}-u_{-}^{3}}{u_{+}-u_{-}}, \quad u_{*}<u_{0}<u_{+} .
$$

The orbit connecting $\left(u_{-}, 0\right)$ to $\left(u_{*}, 0\right)$ is not overcompressive, but fails to satisfy the linear independence condition and therefore is not stable in the linear hyperbolic sense:

$$
\left(u_{*}, 0\right)-\left(u_{-}, 0\right)=c r_{2}\left(u_{*}, 0\right)
$$

for a scalar $c$. Note that (3.4) states the linear dependency of the jump of the shock and the eigenvector corresponding to the characteristic $\lambda_{2}\left(u_{*}, 0\right)$ leaving the shock. The shock $\left(u_{*}, 0 ; u_{+}, 0\right)$ is a Lax shock and has a unique saddle-node connection. 


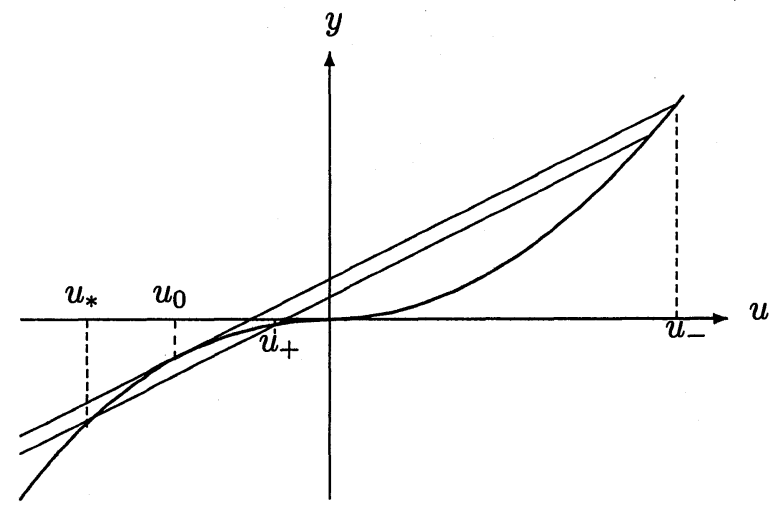

FigURE 3.1

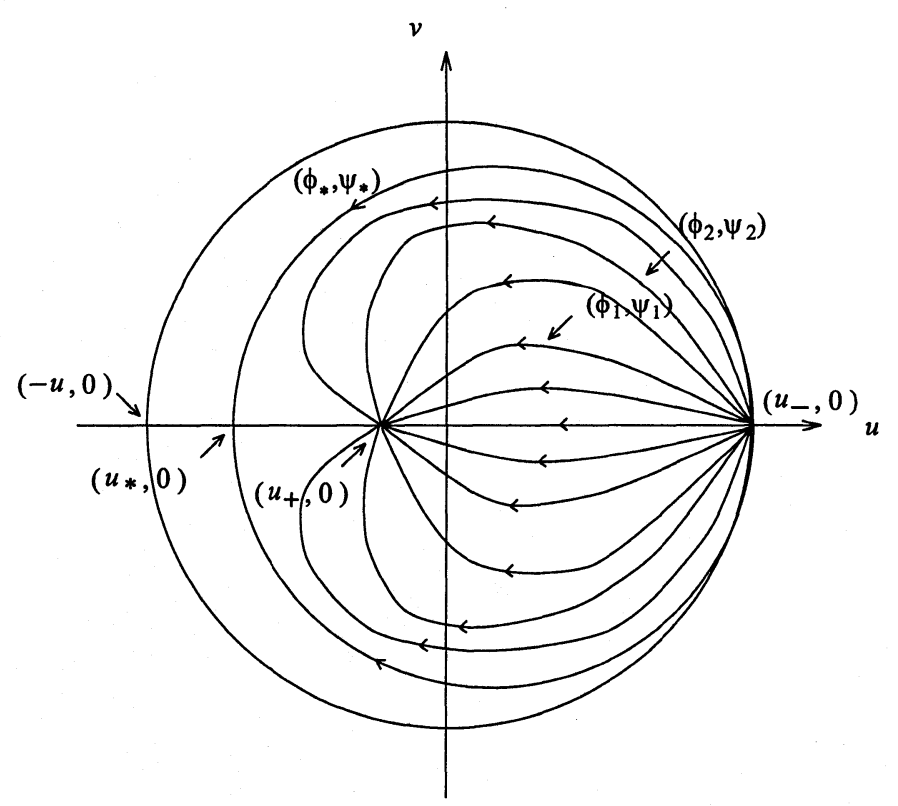

FIGURE 3.2

\section{Nonlinear stability of shock profiles}

We first consider the perturbation of one of the overcompressive shock profiles $\left(\phi_{1}, \psi_{1}\right)$, Figure (3.1):

$$
(u, v)(x, 0)=\left(\phi_{1}, \psi_{1}\right)(x / \varepsilon)+(\bar{u}, \bar{v})(x, 0) .
$$

Since the wave is overcompressive, (3.3), there is no characteristic leaving the shock. Consequently, if the shock is stable, then the perturbation would propagate toward the wave from both sides, and the solution tends to a profile $\left(\phi_{2}, \psi_{2}\right)$, Figure 3.1 , properly translated in phase. Consequently, the convergence would be in a strong sense, in 
particular in $L_{p}(x), 1 \leq p \leq \infty$. From the conservation laws, this implies that

$$
\int_{-\infty}^{\infty}\left((u, v)(x, t)-\left(\phi_{2}, \psi_{2}\right)\left(\left(x+x_{0}-s t\right) / \varepsilon\right)\right) d x=0, \quad t \geq 0
$$

for a translation $x_{0}$ in phase. This is so for small perturbations and fixed viscosity [3]. We now consider the case of vanishing viscosity and fixed perturbation. The translation affects the conservation laws as follows:

$$
\left.\int_{-\infty}^{\infty}\left((\phi, \psi)\left(\left(x+x_{0}-s t\right) / \varepsilon\right)\right)-(\phi, \psi)((x-s t) / \varepsilon)\right) d x=x_{0}\left(u_{+}-u_{-}, 0\right) .
$$

This identity follows by showing that the derivative of the left-hand side with respect to $x_{0}$ is $\left(u_{+}-u_{-}, 0\right)$, as a consequence of (3.1). Thus the translation only affects the first conservation law and does not depend on the viscosity $\varepsilon$. We now study the second conservation law, which is affected only through the change of the profile from $\left(\phi_{1}, \psi_{1}\right)$ to $\left(\phi_{2}, \psi_{2}\right)$. Since these profiles are functions of $\xi=(x-s t) / \varepsilon$, we have from (4.1) and (4.2) that

$$
\int_{-\infty}^{\infty} \bar{v}(x, 0) d x=\varepsilon \int_{-\infty}^{\infty}\left(\psi_{2}-\psi_{1}\right)(\xi) d \xi
$$

The main question of the stability of overcompressive shocks is the following: For a given perturbation $\bar{v}$ of a profile $\psi_{1}$, can another profile $\psi_{2}$ be found to satisfy (4.3)? For this to hold for vanishing viscosity, $\varepsilon \rightarrow 0$, the integral on the right-hand side of (4.3) has to be arbitrarily large for varying $\psi_{2}$. This is not so, as we will see. Note that the profiles $(\phi, \psi)(\xi)$ are independent of the viscosity $\varepsilon,(3.1)$.

Consider the integral for a profile with $\psi(\xi)>0$; profiles in the lower-half plane are similar,

$$
\int_{-\infty}^{\infty} \psi(\xi) d \xi=\int_{-\infty}^{\infty}\left[u_{-}\left(u_{-}^{2}-s\right)\right]^{-1} \psi^{2} d \tau
$$

where we have used (3.1) and changed the integration variable from $\xi$ to $\tau=\phi / \psi$. It follows from (4.4) that an outer profile has larger integral than the inner ones. Thus the integral on the right-hand side of (4.3) is bounded by

$$
M=M\left(u_{*}\right)=2 \int_{-\infty}^{\infty} \psi_{*}(\xi) d \xi
$$

Here we have noted that the constant $M$ is a function of the varying right state $u_{*}$, $-u_{-}<u_{*}<u_{0}$, with the left state $\left(u_{-}, 0\right)$ fixed. This fact will be useful later. We thus conclude that (4.3) cannot be satisfied if

$$
\varepsilon<M^{-1}\left|\int_{-\infty}^{\infty} \bar{v}(x, 0) d x\right|
$$

In other words, the stability is not uniform in the strength $\varepsilon$ of the viscosity. Thus, overcompressive shocks should not be regarded as being admissible for the hyperbolic theory. This is consistent with the study of Section 2 where we have shown that the hyperbolic theory is well-posed only if the overcompressive shocks are excluded. 
We next study the question of what happens when (4.3) fails for any profiles $\left(\phi_{2}, \psi_{2}\right)$. First, we note that along a trajectory of $(3.1)$, we have

$$
\begin{aligned}
\frac{d \phi}{d \xi} & =\phi\left(\phi^{2}+\psi^{2}-s\right)+u_{-}\left(s-u_{-}^{2}\right) \\
\frac{d \psi}{d \xi} & =\psi\left(\phi^{2}+\psi^{2}-s\right) \\
\frac{d\left(\phi^{2}+\psi^{2}\right)}{d \xi} & =2\left(\phi^{2}+\psi^{2}\right)\left(\phi^{2}+\psi^{2}-s\right)+2 u_{-}\left(s-u_{-}^{2}\right) .
\end{aligned}
$$

From these we deduce

$$
\frac{d^{2}\left(\phi^{2}+\psi^{2}\right)}{d \xi}=u_{-}\left(u_{-}^{2}-s\right)\left(u_{-}-\phi\right) \quad \text { whenever } \quad \frac{d\left(\phi^{2}+\psi^{2}\right)}{d \xi}=0 .
$$

We claim from $(4.7 \mathrm{a})-(4.7 \mathrm{~d})$ that along the trajectories connecting $\left(u_{-}, 0\right)$ to either $\left(u_{+}, 0\right)$ or $\left(u_{*}, 0\right)$

$$
\frac{d\left(\phi^{2}+\psi^{2}\right)}{d \xi}<0
$$

We only will consider the trajectories in the upper half plane $\psi>0$. From (4.7b), the trajectories must cross the circle $\phi^{2}+\psi^{2}=s$ in the right-half plane $\phi>0$. Before crossing $\phi^{2}+\psi^{2}=s$, we have from (4.7b) that $d \psi / d \xi<0$. We will show that $\phi^{2}+\psi^{2}$ must be strictly decreasing there. Otherwise, if $\phi^{2}+\psi^{2}$ is stationary, then $d \phi / d \xi<0$ there, and from (4.7d), we conclude that $d^{2}\left(\phi^{2}+\psi^{2}\right) / d \xi>0$. This implies that $\phi^{2}+\psi^{2}$ begins to increase. It cannot become stationary again because this would contradict $d^{2}\left(\phi^{2}+\psi^{2}\right) / d \xi>0$. In other words, the trajectory will not cross the circle $\phi^{2}+\psi^{2}=s$ to eventually connect $\left(u_{+}, 0\right)$ or $\left(u_{*}, 0\right)$.

We next consider the region inside the circle $\phi^{2}+\psi^{2}=s$. In the right-half plane $\phi>0$, it follows from (4.7c) that $\phi^{2}+\psi^{2}$ is decreasing. There are two remaining cases. In the first case $\phi<0, d \phi / d \xi>0$, we have $\phi^{2}+\psi^{2}$ decreasing since $d \psi / d \xi<0$ by (4.7b), and clearly $d|\phi| / d \xi=-d \phi / d \xi<0$. In the final case $\phi<0, d \phi / d \xi<0$, we use (4.7c) and (4.7d) as above to conclude that once $\phi^{2}+\psi^{2}$ becomes stationary then it will increase and not become a connecting orbit. This shows that along any connecting orbit, $\phi^{2}+\psi^{2}$ decreases (4.7e). In particular, the trajectory $\left(\phi_{*}, \psi_{*}\right)$ approaches the circle $u^{2}+v^{2}=u_{-}^{2}$ as $u_{*}$ tends to $-u_{-}$. As $u_{*}$ approaches $-u_{-}$, the speed $s_{*}$ of $\left(u_{-}, 0 ; u_{*}, 0\right)$ tends to $u_{-}^{2}$ and we conclude from the above and $(4.7 \mathrm{~b})$ that

$$
\lim _{u_{*} \rightarrow-u_{-}} M\left(u_{*}\right)=\infty \text {. }
$$

Moreover, using the analysis above, we can see that $M\left(u_{*}\right)$ is a strictly monotone function of $u_{*}$. Assume, without loss of generality, that the integral on the left side of (4.3) is positive. In this case (4.3) fails if and only if

$$
\int_{-\infty}^{\infty} \bar{v}(x, 0) d x>\varepsilon \int_{-\infty}^{\infty}\left(\psi_{*}-\psi_{1}\right)(\xi) d \xi
$$

Thus, overcompressive shocks should not be regarded as being admissible for the hyperbolic theory. This is consistent with the study of Section 2, where we have shown that the hyperbolic theory is well-posed only if the overcompressive shocks are excluded. 
We next study the question of what happens when (4.3) fails for any profiles $\left(\phi_{2}, \psi_{2}\right)$.

From (4.8), there exists a unique $\hat{u}$ between $u_{*}$ and $-u_{-}$such that the trajectory $(\hat{\phi}, \hat{\psi})$ connecting $\left(u_{-}, 0\right)$ and $(\hat{u}, 0)$ with speed $\hat{s}$ has the property that (4.3) holds when $\psi_{2}$ is replaced by $\hat{\psi}$. With this, the solution approaches the combination of two traveling waves: the slower one is $(\hat{\phi}, \hat{\psi})$ connecting $\left(u_{-}, 0\right)$ and $(\hat{u}, 0)$, and the faster one is the Lax shock connecting $(\hat{u}, 0)$ and $\left(u_{+}, 0\right)$. Moreover, as the viscosity $\varepsilon$ tends to zero, the state $\hat{u}$ approaches $-u_{-}$, and the trajectory $(\hat{\phi}, \hat{\psi})$ has non-vanishing shock layer, which corresponds to contact discontinuities in the hyperbolic theory.

We remark that the trajectory $(\hat{\phi}, \hat{\psi})$ does not satisfy the aforementioned linear independence stability criterion and must split into two waves of different speeds when perturbed. This is true even for fixed positive viscosity and arbitrarily small perturbation. The proof of this follows from an analysis similar to that above.

\section{Part II. Undercompressive Shocks}

\section{Inviscid models}

The inviscid combustion model corresponding to (1.2) captures some of the characteristics of the Chapman-Jouguet theory for the reactive compressible Euler equations [1],

$$
\begin{gathered}
(u+q z)_{t}+f(u)_{x}=0 \\
z_{t}=-\delta\left(u-u_{0}\right) z .
\end{gathered}
$$

Here the variable $u$ represents the thermodynamics variable of the gas, and $z$ is the reactant with $z=1$ corresponding to unburnt reactant and $z=0$ corresponding to completely burnt reactant. The constant $q$ is the heat release due to burning, and $u_{0}$ is the ignition temperature. The Dirac delta function in the second equation signifies that the burning takes place instantaneously at the ignition temperature $u_{0}$. To capture detonation waves, we assume that the flux function $f(u)$ satisfies

$$
f^{\prime}(u)>0, \quad f^{\prime \prime}(u)>0 .
$$

With this, the speed $s$ of a combustion wave is positive, and the upstream state is burnt and downstream state is unburnt:

$$
\begin{array}{rlr}
(U, Z)(-\infty) & =\left(u_{-}, 0\right), \quad u_{-}>u_{0} \\
(U, Z)(\infty) & =\left(u_{+}, 1\right), \quad u_{+}<u_{0}, \\
(u, z)(x, t) & =(U, Z)(x-s t) .
\end{array}
$$

It is easy to see from the second equation of $(5.1)$ that $(U, Z)$ is a simple step function, and from the first equation that the following jump condition holds:

$$
\begin{gathered}
(U, Z)(x-s t)= \begin{cases}\left(u_{-}, 0\right), & x-s t<x_{0}, \\
\left(u_{+}, 1\right), & x-s t>x_{0},\end{cases} \\
s\left(u_{+}+q-u_{-}\right)=f\left(u_{+}\right)-f\left(u_{-}\right) .
\end{gathered}
$$

From the jump condition (5.4) we have the following three possibilities:

In the first case the speed $s=s_{*}$ given uniquely by

$$
s_{*}\left(u_{+}+q-u_{*}\right)=f^{\prime}\left(u_{*}\right) \text {. }
$$


We set $u_{-}=u_{*}$ and the combustion wave $\left(5.3^{\prime}\right)$ is called a Chapman-Jouguet detonation. The speed is characteristic with respect to the state behind the wave:

$$
f^{\prime}\left(u_{*}\right)=s_{*}>f^{\prime}\left(u_{+}\right) \text {. }
$$

For speed $s>s_{*}$, we can have either $u_{-}=u_{0}$ or $u_{-}=u^{0}$, cf. Figure 5.1. The wave $\left(u_{0}, 0 ; u_{+}, 1\right)$ is called the weak detonation and $\left(u^{0}, 0 ; u_{+}, 1\right)$ the strong detonation. Weak detonation is undercompressive as compared with the strong detonation, which is compressive as a gas shock:

$$
\begin{gathered}
f^{\prime}\left(u_{+}\right)<f^{\prime}\left(u_{0}\right)<s \\
f^{\prime}\left(u_{+}\right)<s<f^{\prime}\left(u^{0}\right) .
\end{gathered}
$$

Which detonation wave actually occurs in a given flow cannot be determined from the Chapman-Jouguet theory based on the inviscid model (5.1). As we will see in the next section, the question can be settled satisfactorily only when dissipation is taken into consideration.

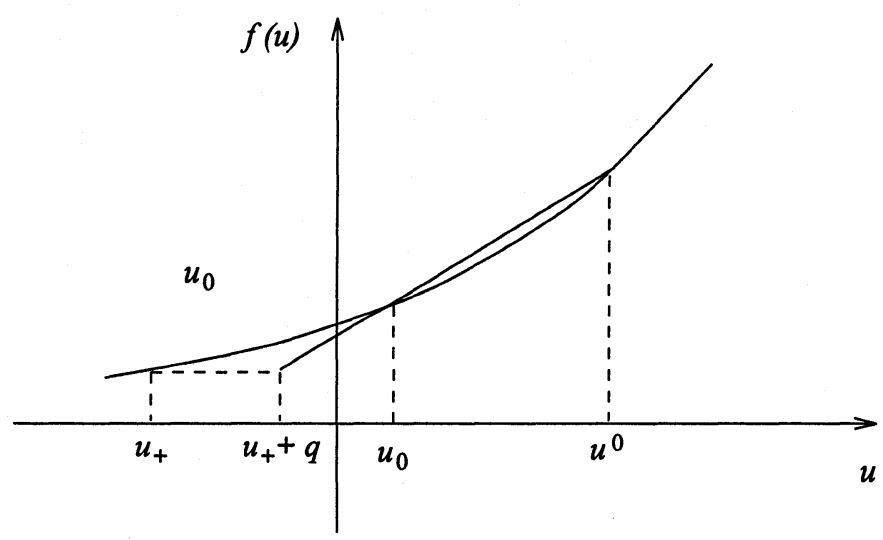

FigURE 5.1

There is another inviscid model where the burning is assumed to be at a finite rate:

$$
\begin{gathered}
(u+q z)_{t}+f(u)_{x}=0 \\
z_{t}=-K \phi(u) z .
\end{gathered}
$$

The function $\phi(u)$ is assumed to be nondecreasing

$$
\phi(u) \begin{cases}=0, & u<0 \\ >0, & u>0 \\ =1 & u>1\end{cases}
$$

and the positive constant $K$ is the reaction rate. This model captures the Zeldovichvon Neumann-Doring (ZND) detonation wave, which is a shock for the gas equation

$$
u_{t}+f(u)_{x}=0
$$

followed by a reaction zone. In fact, due to the lack of heat diffusion, a combustion wave can be started only with a gas shock. We will see that (5.8) is a particular limit of the viscous model (1.2). 


\section{Viscous combustion waves}

Consider the combustion model (1.2) with finite reaction rate and heat conductivity:

$$
\begin{gathered}
(u+q z)_{t}+f(u)_{x}=\varepsilon u_{x x}, \\
z_{t}=-K \phi(u) z .
\end{gathered}
$$

Here the functions $f(u), \phi(u)$ satisfy (5.2) and (5.9), and $\varepsilon$, the heat conductivity, is a positive constant. A combustion wave $\left(u_{-}, 0 ; u_{+}, 1\right)$ satisfies

$$
\begin{gathered}
U^{\prime}=-s(U+q Z)+f(U)+A, \\
Z^{\prime}=-\frac{\beta}{s} \phi(U) Z, \\
(u, z)(x, t)=(U, Z)(\xi), \quad(U, Z)(-\infty)=\left(u_{-}, 0\right), \quad(U, Z)(\infty)=\left(u_{+}, 1\right), \\
\xi=\frac{x-s t}{\varepsilon}, \quad \beta=\varepsilon K A=s u_{-}-f\left(u_{-}\right)=s\left(u_{+}+q\right)-f\left(u_{+}\right) .
\end{gathered}
$$

We have the same jump condition (5.4) and the three possibilities (5.6) for the far field states $\left(u_{-}, 0\right)$ and $\left(u_{+}, 1\right)$. Which of the three possibilities $(5.6)_{c j},(5.6)_{s}$, and $(5.6)_{w}$ actually can occur requires solving the ODE (6.2). From (5.6) and the above, we see that $\left(u_{0}, 0\right)$ is a saddle critical point and $\left(u^{0}, 0\right)$ an unstable node for $(6.2)$. The trajectories for (6.2) are horizontal in the left-half plane $(U, Z), U<0$ because $\phi(u)=0$ there, (5.9). All trajectories leave $\left(u^{0}, 0\right)$ and only one trajectory leaves the saddle point $\left(u_{0}, 0\right)$. The trajectory leaving $\left(u_{0}, 0\right)$ does not reach the right state $\left(u_{+}, 1\right)$, in general. From the second equation of (6.2), we see that $Z^{\prime}(\xi)$ increases when the speed $s$ of the wave decreases. Thus for fixed upstream state $\left(u_{+}, 1\right)$, we have the following two possibilities:

Case I.: There exists a trajectory connecting $\left(u^{0}, 0\right)$ to $\left(u_{+}, 1\right)$ for any speed $s \geq$ $s_{*}$. In other words, the strong detonation is possible up to the Chapman-Jouget detonation with speed $s_{*}$, (5.5), Figure 6.1.

Case II.: There exists $s_{0}>s_{*}$ such that for $s>s_{0}$, strong detonation is possible; for $s=s_{0}$, weak detonation is possible, and for $s<s_{0}$, no combustion wave is possible, Figure 6.2. We denote the states $u_{0}, u^{0}$ with speed $s=s_{0}$ by $u_{1}, u^{1}$.

Note that for the above waves, their existence and shape depend on the various constants. The dependence on the heat conductivity $\varepsilon$ and the reaction rate $K$ is through the constant $\beta=\varepsilon K$. The inviscid model (5.9) carrying the ZND wave is the limit $\varepsilon=0$, and therefore only Case I can occur.

\section{Time-asymptotic states with combustion wave}

In order to understand the nonlinear stability of combustion waves, we now study the large-time behavior of a general solution with given end states

$$
\begin{gathered}
(u, z)(x, t)= \begin{cases}\left(u_{l}, 0\right), & x \rightarrow-\infty, \\
\left(u_{r}, 1\right), & x \rightarrow \infty,\end{cases} \\
u_{l}>0>u_{r} .
\end{gathered}
$$

Waves interact nonlinearly and as the time becomes larger, the interaction dies down, and the solution tends to non-interacting waves. For overcompressive shocks studied in Part I, we identify the time-asymptotic wave patterns through the consideration of conservation laws. Such an analysis does not apply to undercompressive waves because 


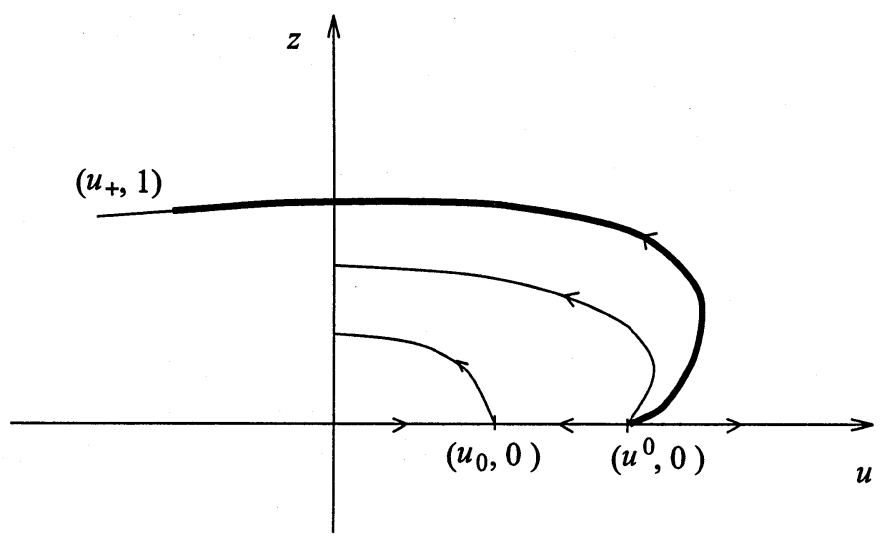

FIGURE 6.1. Strong detonation

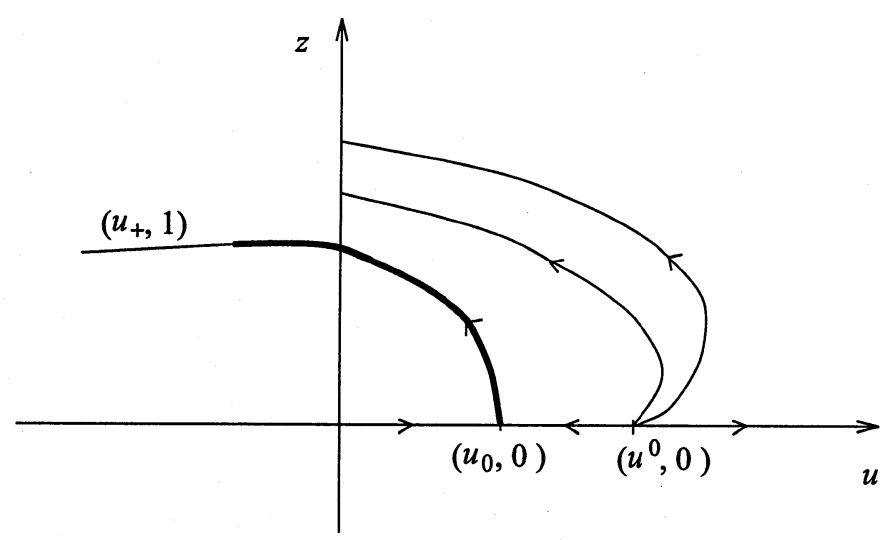

FIGURE 6.2. Weak detonation

the number of outgoing waves exceeds the number of conservation laws. We now study such a time-asymptotic wave pattern of noninteracting waves possibly containing the undercompressive weak detonation wave. If there exists a unique time-asymptotic wave pattern connecting the given end states (7.1), then the waves in the pattern would be nonlinearly stable. We will see that each of the combustion waves, (5.6), can occur generically and is nonlinearly stable in this sense. Let the combustion wave contained in the wave pattern be $\left(u_{-}, 0 ; u_{+}, 1\right)$. The wave in the pattern in front of (behind) the combustion wave is a non-reacting wave of

$$
u_{t}+f(u)_{x}=\varepsilon u_{x x},
$$

and, to be non-interacting with the combustion wave, has speed greater (less) than the combustion speed $s$. Note that any combustion wave is compressive with respect to the state in front of it, (5.6), $s>f^{\prime}\left(u_{+}\right)$. As a consequence, there can exist no wave of (7.2) in front of the combustion wave, so $u_{+}=u_{r}$. Between the combustion wave and the left state $\left(u_{l}, 1\right)$, there may exist non-reacting waves. With the right 
state $\left(u_{+}, 1\right)=\left(u_{r}, 1\right)$ set, we have Case I and Case II of the last section. We consider first Case I. There are two subcases:

Case I1.: $u_{l}>u_{*}$. The time-asymptotic state is a strong combustion wave $\left(u_{l}, 0 ; u_{r}, 1\right)$.

Case I2.: $u_{l} \leq u_{*}$. The time-asymptotic state is the rarefaction wave $\left(u_{l}, u_{*}\right)$ of (7.2) followed by a Chapman-Enskog combustion wave $\left(u_{*}, 0 ; u_{+}, 1\right)$.

For Case II we have three subcases:

Case II1.: $u_{l}>u^{1}$. The time-asymptotic state is a strong detonation wave $\left(u_{l}, 0\right.$; $\left.u_{r}, 1\right)$.

Case II2.: $u_{1}<u_{l}<u^{1}$. The time-asymptotic state consists of a shock $\left(u_{l}, u_{1}\right)$ of (7.2) followed by the weak detonation wave $\left(u_{1}, 0 ; u_{r}, 1\right)$.

Case II3.: $u_{l} \leq u_{1}$. The time-asymptotic state is a rarefaction wave $\left(u_{l}, u_{1}\right)$ of (7.2) followed by the weak detonation wave $\left(u_{1}, 0 ; u_{r}, 1\right)$.

It is easy to show that the above description of time-asymptotic states is complete. For given end states (7.1), there is a unique time-asymptotic state. All three types of combustion waves occur generically. In particular, weak detonations occur generically and are nonlinearly stable. Recall that a weak detonation exists only for particular speeds. Nevertheless, it can occur in a general flow along with other waves, in our situation the non-interacting waves of (7.2). This is typical of undercompressive waves. The nonlinear stability of strong detonation waves have been shown in [13].

Acknowledgements. Research supported in part by Army Research Grant DAAH 04-94-GS0045 and NSF Grant DMS-96-23025.

\section{References}

1. R. Courant and K. O. Friedrichs, Supersonic Flow and Shock Waves, Springer, 1948.

2. H. Freistuhler, Dynamical stability and vanishing viscosity: A case study of a non-strictly hyperbolic system of conservation laws, Comm. Pure Appl. Math. 45 (1992), 561-582.

3. H. Freistuhler and T.-P. Liu, Nonlinear stability of overcompressive shock waves in a rotational invariant system of viscous conservation laws, Comm. Math. Phys. 153 (1993), 147-158.

4. W. Fricket and W. C. Davis, Detonations, University of California Press, 1979.

5. W. Fricket, Introduction to Detonation Theory. University of California Press, Berkeley, 1985.

6. J. Goodman and Z. Xin, Viscous limits for piecewise smooth solutions to systems of conservation laws, Archive Rational Mech. Ana. 121 (1992), 235-265.

7. D. Hoff and T.-P. Liu, The inviscid limit for the Navier-Stokes equations of compressible isentropic flows with a shock data, Indiana J. (1989).

8. A. Jeffereys, Magnetohydrodynamics, Oliver and Boyd, 1966.

9. P. D. Lax, Hyperbolic conservation laws II, Comm. Pure Appl. Math. 101 (1957), 537-566.

10. T.-P. Liu, Riemann problem for general system of hyperbolic conservation laws, J. Diff. Equ. (1975).

11. 1991.

12. _ Pointwise convergence to shock waves for viscous conservation laws, Comm. Pure Appl. Math. 50 (1997), 1113-1182.

13. T.-P. Liu and L. Ying, Nonlinear stability of strong detonations for a viscous combustion model, SIAM J. Math. Anal. 26 (1995), 519-528.

14. T.-P. Liu and K. Zumbrun, On nonlinear stability of general undercompressive viscous shock waves, Comm. Math. Phys. 174 (1995), 319-345.

15. A. Majda, A qualitative model for dynamic combustion, SIAM J. Appl. Math. 41 (1981), 70-91.

16. A. Majda and R. Rosales, Weakly nonlinear detonation waves, SIAM Appl. Math. 43 (1983), 1086-1113.

DePartment of Mathematics, Stanford University, U.S.A. 